\title{
PERCUTANEOUS LUNG BIOPSY: SHOULD IT BE AVOIDED OR NOT?
}

\author{
Cezar I. BETIANU ${ }^{1,2}$, Andrei V. NEAGU1 $₫$, Daniel PANTILE ${ }^{3}$, Ana D. PASCARIU1, \\ Alexandru BAJENARU ${ }^{1}$, Andreea I. NEAGU ${ }^{1}$, Giorgiana ION ${ }^{1}$, Andrei BONDARI ${ }^{4}$ \\ ${ }^{1}$ Department of Radiology, University Emergency Military Central Hospital "Dr. Carol Davila“, Bucharest, \\ Romania \\ ${ }^{2}$ Department of Interventional Radiology, University Emergency Military Central Hospital "Dr. Carol \\ Davila“, Bucharest, Romania \\ ${ }^{3}$ Department of Thoracic Surgery, University Emergency Military Central Hospital "Dr. Carol Davila“, \\ Bucharest, Romania \\ ${ }^{4}$ Radiology and Imaging Department, Faculty of Medicine, University of Medicine and Pharmacy, Craiova, \\ Romania
}

Received 14 Oct 2020, Accepted 05 Nov 2020

https://doi.org/10.31688/ABMU.2020.55.4.08

\section{Abstract}

Introduction. Percutaneous core biopsy (PCB) is a minimally invasive, widely available and relatively safe procedure enabling pathological diagnosis in oncological patients.

The objective of our study was to analyse the complication rates of PCBs between the lungs and other organs, for a better preprocedural evaluation.

Material and methods. We performed a retrospective observational study of 558 PCBs, divided into: lung PCBs $(n=355)$ and other PCBs $(n=203)$. Descriptive statistics, the independent sample t-test, and Pearson correlation coefficient were used to analyse the statistical differences and relationships with the rate of complications.

Results. The largest number of procedures was performed on the lungs $(n=355)$, followed by breast $(n=70)$ and liver $(n=45)$ biopsies. The complication rate was

\section{Résumé}

La ponction-biopsie percutanée pulmonaire : faut-il l'éviter ou non?

Introduction. La ponction-biopsie percutanée (PBP) est une procédure relativement sûre, permettant d'obtenir un diagnostic pathologique chez les patients oncologiques.

Objectif. Étant donné que les biopsies pulmonaires ont un taux de complications plus élevé, on doit se poser la question s'il est meilleur d'éviter celles-ci dans les cas où il s'agit de lésions localisées au niveau de plusieurs organes.

Matériel et méthodes. Il s'agit d'une étude observationnelle rétrospective portant sur 558 PCB réparties en : PBP pulmonaires $(n=355)$ et PBP d'autres organes $(\mathrm{n}=203)$. Les statistiques descriptives, le test $t$ de l'échantillon indépendant et le coefficient de corrélation de 
$7.7 \%(n=43)$. The lung had a higher rate of complications $(11.3 \%$; $n=40)$, compared to $1.5 \%(n=3)$ from other PCBs. Lung PCBs correlate with complications $(\mathrm{r}(556)=.18, \mathrm{p}<.001)$. The independent samples t-test was statistically significant, $\mathrm{t}(501)=5.2, \mathrm{p}<001$. The lung PCBs were associated with a statistically significantly larger mean complication occurrence than the other PCBs. Cohen's d was estimated at .37, which is a medium effect. The most common complication was pneumothorax $(n=31)$, rate of $8.1 \%(n=29)$ from lung biopsies. CT-guidance $(\mathrm{r}(487)=.15, \mathrm{p}=.001)$, repeated biopsies $(\mathrm{r}(538)=.17, \mathrm{p}<.001)$, and INR $(\mathrm{r}(524)=.10, \mathrm{p}$ $=.019)$ are correlated with complications.

Conclusions. Lung biopsy is riskier than other biopsies and should be avoided if another biopsy, in the same pathological context, is available, to avoid unnecessary complications.

Keywords: pneumothorax, haemothorax, ultrasound, computer tomography, image-guided

\author{
Abbreviations \\ $\mathrm{PCB}=$ percutaneous core biopsy; \\ US = ultrasound; \\ $\mathrm{CT}=$ computed tomography; \\ $\mathrm{SD}=$ standard deviation; \\ PET-CT = positron emission tomography-computed \\ tomography; \\ MRI = magnetic resonance imaging; \\ INR = international normalized ratio.
}

\section{INTRODUCTION}

Lung percutaneous core biopsy (PCB) is a minimally invasive technique with increasingly wide-spread use due to its wide availability, cost-effectiveness, and safety. This technique can influence the therapeutic strategy and lung disease staging, by improving the histopathological diagnosis ${ }^{1}$. Needle gauge size or imaging guidance do not influence its high diagnostic accuracy. However, its precise placement in the diagnostic algorithm is still debatable, being considered a complementary procedure ${ }^{2}$.

Technical improvements like imaging guidance or needle design have contributed to decreasing the rate of technical failure, the rate of complications, and especially major complications (those in need of intervention). They also increased the accuracy, making lung PCB a ubiquitous method ${ }^{3}$ Of note is imaging guidance that sustained significant technical improvements, making needle placement safer and faster. There are two categories of imaging guidance: ultrasonography (US) and computed tomography (CT). CT allows a full anatomical view and permits trajectories that avoid
Pearson ont analysé les différences statistiques et les relations avec l'apparition des complications.

Résultats. Le taux de complications global a été de $7,7 \%$. Le taux de complication le plus élevé a été rencontré chez les patients soumis aux PBP pulmonaires $11,3 \%$, tandis que les PBP à d'autres localisations ont enregistré un taux de complications de 1,5. La moyenne de complications des PBP pulmonaires a été statistiquement significativement plus élevée que les PBP des autres organes. La complication la plus fréquente a été le pneumothorax, dont la majorité est survenue au cours de PBP pulmonaires. Les PBP guidées par scanner $(\mathrm{r}(487)=.15, \mathrm{p}=.001)$, les biopsies répétées $(\mathrm{r}(538)=.17, \mathrm{p}<.001)$ et la valeur d'INR ( $\mathrm{r}(524)=.10$, $\mathrm{p}=.019)$ sont corrélées aux complications.

Conclusions. Pour éviter des complications inutiles, si le contexte pathologique le permet, il est recommandé a choisir pour la PBP une autre localisation sauf celle pulmonaire.

Mots-clés: pneumothorax, hémothorax, hémorragie, échoguidée, tomodensitométrie, sous guidage.

anatomical structures, providing possible access to central lesions. The most significant drawbacks of this technique are the duration of the procedure and the radiation dose; CT fluoroscopy improved only the first one $e^{4}$. The US offers real-time imaging and shorter procedure time, but in lung it is limited to the peripheral lesions, thus having a lower complication rate. It is preferable to use US-guidance whenever possible ${ }^{5-7}$.

As a minimally invasive procedure, it has potential complications. Although studies report low rates, they are higher compared to other biopsy techniques Within these low rates, it stands out the comparatively higher rates of lung biopsies. Complication occurrence can prolong hospital stay and decrease the cost-effectiveness of the procedure. Complications for lung PCBs are reported with rates up to five times higher ${ }^{8}$.

Appropriate preprocedural planning is needed to minimize the risks associated with lung PCBs. In cases of multiple potential lesions located in more than one organ, part of the procedural planning is to choose which lesion to puncture. The most feasible 
lesion is the one in which the possible benefits outweigh the risks. Considering that lung biopsies have a higher complication rate reported, it is worth considering if, in cases with lesions in multiple organs, it should be avoided ${ }^{8}$.

The objective of THE STUDY was to analyse the complication rates of PCBs between the lungs and other organs, for a better preprocedural evaluation.

\section{Material AND Methods}

\section{Sampling Procedures}

This is a retrospective, single-center study, involving patients who underwent CT-guided and US-guided PCBs, in the Department of Interventional Radiology of the University Emergency Military Central Hospital "Dr. Carol Davila", Bucharest, Romania, between March 2015 and October 2018.

The study is approved by the hospital's ethics committee. The patients were informed about the possible complications and their specific conditions and signed a written consent before every procedure, including the processing of personal data for medical purposes.

For this study, we did not implement a systematic sampling plan. Oncologists or other clinicians recommended the patients to our clinic, where an attending radiologist performed image analysis to confirm the diagnostic suspicion and indication for biopsy. The same radiologist evaluated the needle pathway considering the safest track and the accuracy. The patient was required to have recent preprocedural CT/PET-CT/MRI images available. If not, a preprocedural CT was necessary. The CT scanner and ultrasound machine used in the study were Phillips Brilliance 64 and GE LOGIQ P9, respectively.

If the patient did not have coagulation disorders, anticoagulation treatment, or any condition that could affect the outcome, PCB was performed.

The attending radiologist chose the needle characteristics, gauge size, automated/semiautomated, according to his preference. The difficulty of the case or the anatomical position of the lesion were factors of decision for imaging guidance. CT-guidance assessed the more difficult cases, because CT can visualize complications from the pulmonary and abdominal regions more precisely.

All biopsies were performed using the free-hand technique. US-guided biopsies in which the radiologist did not feel safe were re-evaluated to attempt the possibility of a CT-guided biopsy. After sufficient number of tissue samples were acquired, the patients were kept under observation for at least 2 hours.

\section{Inclusion and exclusion}

A total of 638 procedures were indicated, from which 45 were cancelled because of laboratory findings or patient's refusal. From the remaining 593, we excluded 35 procedures that were not PCBs (drainages and breast markings). Thus, the study included 558 PCB performed in 535 patients (mean age 62.8 years, SD 11.7, age range 19-91 years).

\section{Participant's characteristics}

There were 335 men (62.6\%) with a mean age of 64.2 years, SD 11.1, and an age range between 19-89 years, and 200 women $(37.4 \%$ ) with a mean age of 60.4 years, SD 12.4 and an age range between 24-91 years.

The study was done in an outpatient setting. The patients underwent postprocedural investigations for complications assessment. Patients at high-risk for complications were tightly managed and were transferred to an inpatient setting, if necessary. In our practice, we used $1 \%$ or $2 \%$ lidocaine as local anaesthesia. No sedation or general anaesthesia were required.

\section{Data collection}

Patient's demographics (age, gender), clinical information (about smoking status, obesity, diabetes mellitus, high blood pressure), international normalized ratio (INR), blood glucose levels, and procedure-related information (if it was repeated before, biopsied organ, procedure type, imaging guidance, needle gauge size, complications, interventions, histopathological result) were recorded. We counted complications as mild if no intervention was required and moderate to severe if it was required.

\section{Statistical method}

Data were analysed with IBM SPSS Statistics (Version 20). To compare the relationship between complications and different biopsied organs, we filtered the study population by biopsied organs and divided them into two groups: the lung PCBs and the other PCBs.

The independent sample t-test analysed statistical differences between the two groups for complications and other clinical and procedure-related information.

Descriptive statistics were used to assess the number of complications and the rate for each organ and Pearson product-moment correlation coefficient used to describe the strength of the relationship between organs and complications. Also, correlation analysis was used to explore the relation of complications and intervention upon complications with clinical and procedure-related information. 


\section{Results}

The majority of procedures was performed on the lungs $(n=355)$, followed by breast $(n=70)$ and liver $(n=45)$ biopsies (Table 1$)$. We have divided the cases into two groups, of lung PCBs $(n=355)$ and other PCBs $(n=203)$.
Forty-three postprocedural complications occurred, with a total rate of $7.7 \%$. The lung PCBs had the most complications, 40 , and a rate of $11.3 \%$ (complications/organ PCBs). The complication rate for the other PCBs was $1.5 \%$, with only 3 complications, one for liver, one for breast, and one for mediastinum PCBs. The complication rate for the mediastinum

Table 1. Complication rate and their correlation to every biopsied organ.

\begin{tabular}{|c|c|c|c|c|c|c|}
\hline & \multicolumn{2}{|c|}{ PCBs } & \multicolumn{4}{|c|}{ Complications } \\
\hline & $\mathrm{N}$ & $\%$ & $\mathrm{~N}$ & $\%$ & $r$ & $p$ \\
\hline Lymph node & 10 & $1.8 \%$ & 0 & $0.0 \%$ & -0.04 & 0.357 \\
\hline Digestive & 1 & $0.2 \%$ & 0 & $0.0 \%$ & -0.01 & 0.773 \\
\hline Liver & 45 & $8.1 \%$ & 1 & $2.2 \%$ & -0.06 & 0.151 \\
\hline Breast & 70 & $12.5 \%$ & 1 & $1.4 \%$ & $-0.09^{*}$ & 0.035 \\
\hline Mediastinum & 10 & $1.8 \%$ & 1 & $10.0 \%$ & 0.01 & 0.784 \\
\hline Bone & 33 & $5.9 \%$ & 0 & $0.0 \%$ & -0.07 & 0.087 \\
\hline Soft Tissue & 20 & $3.6 \%$ & 0 & $0.0 \%$ & -0.06 & 0.189 \\
\hline Peritoneal & 2 & $0.4 \%$ & 0 & $0.0 \%$ & -0.02 & 0.683 \\
\hline Lung & 355 & $63.6 \%$ & 40 & $11.3 \%$ & $0.18^{* *}$ & $<0.001$ \\
\hline Renal & 2 & $0.4 \%$ & 0 & $0.0 \%$ & -0.02 & 0.683 \\
\hline Retroperitoneal & 7 & $1.3 \%$ & 0 & $0.0 \%$ & -0.03 & 0.443 \\
\hline Splenic & 1 & $0.2 \%$ & 0 & $0.0 \%$ & -0.01 & 0.773 \\
\hline Adrenal & 2 & $0.4 \%$ & 0 & $0.0 \%$ & -0.02 & 0.683 \\
\hline Total & 558 & & 43 & $7.7 \%$ & & \\
\hline
\end{tabular}

${ }^{*}$ Correlation is significant at the 0.05 level (2-tailed).

${ }^{* *}$ Correlation is significant at the 0.01 level (2-tailed).

Table 2. Correlations between complication occurrence and different clinical and procedural variables.

\begin{tabular}{ccccccc}
\hline & \multicolumn{3}{c}{ Complications } & \multicolumn{3}{c}{ Intervention Upon Complications } \\
\cline { 2 - 7 } & $r$ & $p$ & $N$ & $r$ & $p$ & $N$ \\
\hline Age & 0.04 & 0.321 & 556 & 0.00 & 0.914 & 541 \\
\hline Imaging guidance & $0.15^{* *}$ & 0.001 & 489 & 0.07 & 0.113 & 485 \\
\hline Needle gauge & 0.09 & 0.101 & 356 & 0.04 & 0.483 & 347 \\
\hline $\begin{array}{c}\text { Intervention upon complica- } \\
\text { tions }\end{array}$ & $0.49^{* *}$ & $<0.001$ & 541 & - & - & - \\
\hline Histopathological diagnosis & $-0.17^{* *}$ & $<0.001$ & 524 & -0.08 & 0.067 & 507 \\
\hline Repeated Procedure & $0.17^{* *}$ & $<0.001$ & 540 & $0.10^{*}$ & 0.024 & 540 \\
\hline Smoker status & 0.01 & 0.888 & 143 & 0.07 & 0.376 & 142 \\
\hline Obesity status & -0.08 & 0.434 & 92 & 0.08 & 0.454 & 92 \\
\hline Diabetes mellitus & 0.02 & 0.697 & 423 & 0.06 & 0.261 & 417 \\
\hline High blood pressure & -0.05 & 0.448 & 212 & 0.09 & 0.197 & 209 \\
\hline INR & $0.10^{* *}$ & 0.019 & 526 & -0.07 & 0.116 & 517 \\
\hline Glycemia & 0.03 & 0.563 & 517 & 0.04 & 0.332 & 508 \\
\hline Pneumothorax & - & - & - & $0.47^{* *}$ & $<0.001$ & 541 \\
\hline Haemothorax & - & - & - & $0.14^{* *}$ & 0.001 & 541 \\
\hline Superficial Hematoma & - & - & - & $0.30^{* *}$ & $<0.001$ & 541 \\
\hline Haemorrhage/Haemoptysis & - & - & - & -0.02 & 0.664 & 541 \\
\hline Infection & - & - & - & $0.30^{* *}$ & $<0.001$ & 541 \\
\hline Cor & 1 & & & & \\
\hline
\end{tabular}

*Correlation is significant at the 0.05 level (2-tailed).

${ }^{* *}$ Correlation is significant at the 0.01 level (2-tailed). 
biopsies stands out as $10 \%$, one reason being the low number of 10 mediastinum PCBs.

A Pearson product-moment correlation coefficient was computed to assess the relationship between the type of organ biopsied and postprocedural complications. The results indicated a relatively typical positive relationship between lung $\mathrm{PCB}$ and complications occurrence $(r(556)=0.18, p<0.001)$, and a small inverse relationship between breast PCBs and complications occurrence $(r(556)=-0.089, p=$ $0.035)$. No other significant association was found between complications occurrence and PCBs for other organs.

The most common complication was pneumothorax, with 31 occurrences, 29 cases (8.1\%) from lung biopsies, and one each for mediastinum and liver biopsy (0.9\%).

Haemorrhage/haemoptysis appeared in 9 lung PCBs $(2.5 \%)$, followed by haemothorax in 4 lung PCBs (1.1\%), infection in one lung PCB (0.3\%), and superficial hematoma in one breast PCB (0.5\%). No patient suffered from an air embolism. There were 3 cases with multiple complications, all after lung biopsies. The complication type rate was calculated by the group.

Pearson product-moment correlation coefficient was used to examine the relationship of postprocedural complications and interventions with the clinical and procedural variables (Table 2). The results indicated small positive correlations of complication occurrence with the use of CT guidance $(r(487)=$ $0.15, \mathrm{p}=0.001)$ and repeated procedures $(\mathrm{r}(538)=$ $0.17, \mathrm{p}<0.001$ ); small negative correlations with histopathological diagnosis $(\mathrm{r}(522)=-0.17, \mathrm{p}<0.001)$ and with INR $(r(524)=-0.10, p=0.019)$. A correlation between complications and interventions was expected and it was observed a relatively large one $(\mathrm{r}(339)=0.49, \mathrm{p}<0.001)$. No other statistically significant correlations were observed. In general, post procedural complications are strongly correlated with interventions. CT-guidance, repeated procedures and a low INR correlate with complications occurrence, and complications occurrence is correlated with a failed histopathological diagnosis, although statistically insignificant.

Small positive correlations of intervention upon complication with repeated procedures $(r(548)=$ $0.10, p=0.024)$ and haemothorax $(r(539)=0.140, p=$ $0.001)$ were observed.

Superficial hematoma $(r(539)=0.30, p<0.001)$, infection $(\mathrm{r}(539)=0.30, \mathrm{p}<0.001)$ and pneumotho$\operatorname{rax}(\mathrm{r}(472)=0.47, \mathrm{p}<0.001)$ are strongly correlated with interventions upon complication. Overall, the results support our prediction that complications of lung PCBs have a strong relationship with intervention upon complication.

We elaborated on the correlation between imaging guidance and complications and conducted descriptive analysis and Pearson's correlation coefficient between the two, sorting by the PCB group (Table 3). Results point out a higher frequency of complications in both groups when CT-guidance is used. The lung PCBs have a rate of $13.2 \%(n=38)$ compared to only $2.1 \%(n=1)$ in the other PCBs group. Pearson's coefficient shows a small positive correlation between the two variables in the lung group $(\mathrm{r}(341)=0.11, \mathrm{p}$ $=0.039)$ and no statistically significant correlation in the other group ${ }^{9.11}$.

The lung PCBs group $(n=355)$ was associated with a complication occurrence $\mathrm{M}=0.11(\mathrm{SD}=0.32)$. By comparison, the other PCBs group $(n=203)$ was associated with a smaller complications' occurrence $\mathrm{M}=0.01(\mathrm{SD}=0.12)$. To test the hypothesis that the lung PCBs and other PCBs were associated with statistically significantly different mean complication occurrence, an independent samples t-test was performed. The assumption of homogeneity of variances was tested and did not satisfy via Levene's $F$ Test, $F(556)=87, p<0.001$. The independent samples $\mathrm{t}$-test was associated with a statistically significant

Table 3. Correlation between imaging guidance and complication sorted by PCB type.

\begin{tabular}{|c|c|c|c|c|}
\hline & & & \multicolumn{2}{|c|}{ Complications } \\
\hline & & & Lung PCBs & Other PCBs \\
\hline \multirow{4}{*}{ Imaging guidance } & \multirow{2}{*}{ US } & $\mathrm{N}$ & 2 & 2 \\
\hline & & $\%$ & $3.6 \%$ & $2 \%$ \\
\hline & \multirow{2}{*}{ CT } & $\mathrm{N}$ & 38 & 1 \\
\hline & & $\%$ & $13.2 \%$ & $2.1 \%$ \\
\hline \multirow{3}{*}{\multicolumn{2}{|c|}{ Pearson's correlation }} & $\mathrm{r}$ & $0.11^{*}$ & 0.00 \\
\hline & & $\mathrm{p}$ & 0.039 & 0.987 \\
\hline & & $\mathrm{N}$ & 343 & 146 \\
\hline
\end{tabular}

${ }^{*}$ Correlation is significant at the 0.05 level (2-tailed). 
Table 4. Compared means of clinical and procedural variables between the two groups.

\begin{tabular}{|c|c|c|c|c|c|c|c|c|}
\hline & \multicolumn{2}{|c|}{ Lung } & \multicolumn{2}{|c|}{ Other } & \multirow[b]{2}{*}{$t$} & \multirow[b]{2}{*}{$d f$} & \multirow[b]{2}{*}{$p$} & \multirow[b]{2}{*}{ Cohen's d } \\
\hline & M & $S D$ & M & SD & & & & \\
\hline Complications* & 0.1 & 0.3 & 0.01 & 0.1 & 5.2 & 501 & $<0.001$ & 0.37 \\
\hline Gender* & 1.3 & 0.4 & 1.5 & 0.5 & -6.47 & 383 & $<0.001$ & -0.59 \\
\hline Age $^{*}$ & 65 & 10.1 & 59.3 & 13.3 & 5.28 & 333 & $<0.001$ & 0.5 \\
\hline Imaging guidance* & 0.8 & 0.4 & 0.3 & 0.5 & 11.59 & 224 & $<0.001$ & 1.26 \\
\hline Needle gauge* & 17.9 & 0.4 & 17.6 & 1.6 & 2.42 & 160 & 0.01 & 0.30 \\
\hline Intervention upon complications ${ }^{*}$ & 0.03 & 0.2 & 0.01 & 0.1 & 2.27 & 516 & 0.02 & 0.17 \\
\hline Smoker status* & 0.9 & 0.3 & 0.7 & 0.5 & 1.57 & 21 & 0.13 & 0.49 \\
\hline Obesity status* & 0.3 & 0.4 & 0.5 & 0.5 & -1.93 & 50 & 0.06 & -0.45 \\
\hline Diabetes mellitus ${ }^{* *}$ & 0.2 & 0.4 & 0.2 & 0.4 & -0.45 & 421 & 0.65 & -0.05 \\
\hline High blood pressure ${ }^{* *}$ & 0.7 & 0.5 & 0.7 & 0.5 & 0.01 & 210 & 0.99 & 0 \\
\hline $\mathrm{INR}^{* *}$ & 1.1 & 0.1 & 1.1 & 0.1 & -0.01 & 524 & 0.99 & 0 \\
\hline Glycemia** & 111.4 & 41 & 109.2 & 39.9 & 0.6 & 515 & 0.55 & 0.06 \\
\hline Systolic blood pressure* & 131.1 & 13.3 & 127.7 & 20 & 0.81 & 33 & 0.43 & 0.22 \\
\hline
\end{tabular}

*Equal variances not assumed.

** Equal variances assumed.

effect, $t(501)=5.2, p<0.001$. Thus, the lung PCBs were associated with a statistically significant larger mean complication occurrence than the other PCBs. Cohen's $d$ was estimated at 0.37 , which is a medium effect $^{12}$.

\section{Discussion}

Although it is a well-established diagnostic procedure, with a relatively few complications, lung PCB has a statistically higher complication rate compared to other biopsies. There are studies reporting complication rates of biopsies of lung and other organs separately. However, to our knowledge, there are no studies to compare the complication rates between the lung and other organs ${ }^{13,14}$.

The complication rate of lung biopsies obtained in this study is $11.3 \%$, statistically higher than other biopsies, that had a complication rate of $1.5 \%{ }^{15}$. The moderate-major (those in need of intervention) complication rate difference between the two groups is statistically significant, with a rate of $2.8 \%$ for lung biopsies and a rate of $0.5 \%$ for the other group. In our study, the most frequent complication was pneumothorax, with a rate of $8.1 \%$ from lung biopsies and $0.9 \%$ from other biopsies. Pneumothorax, as a moderate-major complication, had a rate of $2.5 \%$, with 9 cases out of 31 who necessitated tub placement ${ }^{16}$. Other complications that required intervention were haemothorax $(n=1)$ and infection $(n=1)$ for lung biopsies and superficial hematoma $(n=1)$ for the other group. The second most frequent complication was haemorrhage ( $n=9$, respectively $2.5 \%$ ).
The complication rates for both groups were similar with those reported in the literature. Previous studies and meta-analysis related to PCBs and their complications reported pneumothorax and haemorrhage/haemoptysis as common complications. Pneumothorax has a rate of around $20 \%$, with a $6.6 \%$ rate of tube placement. Haemorrhage is encountered in $1 \%$ of cases, and only $0.1 \%$ required intervention ${ }^{17-19}$.

From our experience, the key point that relates to the higher complication rate is CT-guidance use, which is used in instances where the lesion is located more centrally; thus, more pleural surfaces are penetrated, and also the lesion is near-by bigger blood vessels, similar with other studies ${ }^{20}$.

Our study has several limitations that should be mentioned. The analysis was conducted retrospectively; thus, the selection of patients was not randomized between the two groups and between the CT and US guidance. The choice was done by rational decisions made by the attending radiologist. The groups have statistically different means of age, gender, imaging guidance, and needle gauge. Another limitation is the lack of an accurate lesion description or the lack of a thorough patient history, which could influence the complication rate. We did not perform a follow-up to check for tumour seeding. The number of fragments and needle passes per lesion was not recorded; this is a controversial parameter, as multiple punctures could promote or not the occurrence of complications. However, we expect to have the same systematic error in both groups of patients.

The patients who experienced complications were more likely to need intervention, had an 
extended hospitalization, and implicitly a higher hospital cost according to literature reports ${ }^{5}$. We did not record hospital admission. The problem complicating the outpatient management is not the complication, but the need for intervention.

Lesion size, depth, and emphysema along the needle pathway are the main factors influencing the incidence of pneumothorax but were not recorded in our study ${ }^{21}$. More attention should be paid to patients with risk factors for pneumothorax after lung biopsy. It would be useful to evaluate in another study these risk factors when performing a lung biopsy, as it seems that older age, male sex, and chronic obstructive pulmonary disease are the main risk factors for severe pneumothorax ${ }^{22}$.

\section{Conclusions}

Although it is a widely accepted procedure, with a relatively few complications, lung biopsies have a statistically higher complication rate compared to other biopsies. In conclusion, lung biopsy is riskier and more expensive than biopsies performed on other organs. The risk is especially higher when performed under CT-guidance. It should be avoided if another biopsy, in the same pathological context, is available. The lung lesion needs precise planning and detailed knowledge, to avoid unnecessary complications.

\section{Author Contributions:}

Conceptualization, C.I.B and Andrei, B.; methodology, A.V.N.; software, D.P; validation, A.D.P. and A.I.N.; formal analysis, G.I.; investigation, A.B.; resources, C.I.B.; data curation, A.V.N. and G.I.; writing-original draft preparation, A.V.N., G.I., A.D.P., A.I.N. and A.B.; writing-review and editing, C.I.B., Andrei, B. and D.P.; visualization, A.V.N., G.I., A.D.P., A.I.N. and A.B.; supervision, C.I.B. and D.P.; project administration, Andrei, B. All the authors have read and agreed with the final version of the article.

\section{Compliance with Ethics Requirements:}

„The authors declare no conflict of interest regarding this article"

"The authors declare that all the procedures and experiments of this study respect the ethical standards in the Helsinki Declaration of 1975, as revised in 2008(5), as well as the national law. Informed consent was obtained from all the patients included in the study"

"No funding for this study"

\section{Acknowledgements:}

None

\section{References}

1. Wallace MJ, Krishnamurthy S, Broemeling LD, et al. CT-guided percutaneous fine-needle aspiration biopsy of small $(\leq 1-\mathrm{cm})$ pulmonary lesions. Radiology. 2002; 225(3):823-8.

2. Gupta S, Wallace MJ, Cardella JF, Kundu S, Miller DL, Rose SC. Quality improvement guidelines for percutaneous needle biopsy. J Vasc Interv Radiol. 2010; 21(7):969-75.

3. Callister MEJ, Baldwin DR, Akram AR, et al. British Thoracic Society guidelines for the investigation and management of pulmonary nodules: accredited by NICE. Thorax. 2015; 70(Suppl 2):ii1-54.

4. Laurent F, Montaudon M, Latrabe V, Bégueret $\mathrm{H}$. Percutaneous biopsy in lung cancer. Eur J Radiol. 2003; 45(1):60-8.

5. Yang P-C, Chang D-B, Yu C-J, et al. Ultrasound-guided core biopsy of thoracic tumors. Am Rev Respir Dis. 1992; 146(3):763-7.

6. Guo Y-Q, Liao X-H, Li Z-X, et al. Ultrasound-guided percutaneous needle biopsy for peripheral pulmonary lesions: diagnostic accuracy and influencing factors. Ultrasound Med Biol. 2018; 44(5):1003-11.

7. Jeon KN, Bae K, Park MJ, et al. US-guided transthoracic biopsy of peripheral lung lesions: pleural contact length influences diagnostic yield. Acta Radiol. 2014; 55(3):295-301.

8. Gobien RP, Bouchard EA, Gobien BS, Valicenti JF, Vujic I. Thin needle aspiration biopsy of thoracic lesions: impact on hospital charges and patterns of patient care. Radiology. 1983; 148(1):65-7.

9. Al Knawy B, Shiffman M. Percutaneous liver biopsy in clinical practice. Liver Int. 2007; 27(9):1166-73.

10. Abdi W, Millan JC, Mezey E. Sampling variability on percutaneous liver biopsy. Arch Intern Med. 1979; 139(6):667-9.

11. Rao R, Lilley L, Andrews V, Radford L, Ulissey M. Axillary staging by percutaneous biopsy: sensitivity of fine-needle aspiration versus core needle biopsy. Ann Surg Oncol. 2009; 16(5):1170-5.

12. Cohen J. A power primer. Psychol Bull. 1992; 112(1):155.

13. Gignac GE, Szodorai ET. Effect size guidelines for individual differences researchers. Pers Individ Dif. 2016; 102:74-8.

14. Institute for Health Metrics and Evaluation (IHME). GBD Compare Data Visualization. Seattle, WA: IHME, University of Washington. 2017

15. Winokur RS, Pua BB, Sullivan BW, Madoff DC. Percutaneous lung biopsy: technique, efficacy, and complications. In: Seminars in Interventional Radiology. Thieme Medical Publishers; 2013, p. 121-7.

16. Boskovic T, Stanic J, Pena-Karan S, et al. Pneumothorax after transthoracic needle biopsy of lung lesions under CT guidance. J Thorac Dis. 2014; 6(Suppl 1):S99

17. Wiener RS, Schwartz LM, Woloshin S, Welch HG. Population-based risk for complications after transthoracic needle lung biopsy of a pulmonary nodule: an analysis of discharge records. Ann Intern Med. 2011; 155(3):137-44.

18. Heerink WJ, de Bock GH, de Jonge GJ, Groen HJM, Vliegenthart R, Oudkerk M. Complication rates of CT-guided transthoracic lung biopsy: meta-analysis. Eur Radiol. 2017; 27(1):138-48.

19. Yu C-J, Yang P-C, Chang D-B, et al. Evaluation of ultrasonically guided biopsies of mediastinal masses. Chest. 1991; 100(2):399-405.

20. Anderson JM, Murchison J, Patel D. CT-guided lung biopsy: factors influencing diagnostic yield and complication rate. Clin Radiol. 2003; 58(10):791-7. 
21. Lee SM, Park CM, Lee KH, Bahn YE, Kim JI, Goo JM. $\mathrm{C}$-arm cone-beam CT-guided percutaneous transthoracic needle biopsy of lung nodules: clinical experience in 1108 patients. Radiology. 2014; 271(1):291-300.
22. Yang BR, Kim MS, Park CM, Yoon SH, Chae KJ, Lee J. Patterns of percutaneous transthoracic needle biopsy (PTNB) of the lung and risk of PTNB-related severe pneumothorax: A nationwide population-based study. PLoS One. 2020;15(7):e0235599 